\title{
Juvenile Recurrent Parotitis and Sialolithiasis: An Noteworthy Co-Existence
}

\author{
Venkata NR* and Sanjay H \\ Department of ENT and Head \& Neck Surgery, Kohinoor Hospital, India
}

*Corresponding author: Nataraj Rajanala Venkata, Department of ENT and Head \& Neck Surgery, Kohinoor Hospital, Kurla (W), Mumbai, India, Tel: +918691085580; Email: nataraj.rv@gmail.com

\section{Case Report}

Volume 3 Issue 1

Received Date: April 20, 2018

Published Date: May 21, 2018

DOI: $10.23880 /$ ooaj-16000168

\section{Abstract}

Juvenile Recurrent Parotitis is a relatively rare condition. Sialolithiasis co-existing along with Juvenile Recurrent Parotitis is an even rarer occurrence. We present a case of Juvenile Recurrent Parotitis and Sialolithiasis in a 6 years old male child and how we managed it.

Keywords: Juvenile Recurrent Parotitis; Parotid gland; Swelling; Sialolithiasis

\section{Introduction}

Juvenile Recurrent Parotitis is characterized by recurring episodes of swelling usually accompanied by pain in the parotid gland. Associated symptoms usually include fever and malaise. It is most commonly seen in children, but may persist into adulthood. Unlike parotitis, which is caused by infection or obstructive causes like calculi, fibromucinous plugs, duct stenosis and foreign bodies; Juvenile Recurrent Parotitis is usually due to nonobstructive sialectasis. This case demonstrates an unique situation where a Juvenile Recurrent parotitis was found to be associated with sialolithiasis.

\section{Case Report}

A 6 years old male child was brought to us, by his mother, with complaints of recurrent swelling and pain over left parotid area, since past 10 months. The inflammation was responsive to antibiotics but would recur without any predisposing factors. Parotitis was usually accompanied by low grade fever and malaise. As the recurrences started occurring at increasingly frequent occasions, the mother sought medical attention for the child. Tuberculosis was suspected but the tests yielded no results. Even MRI of the parotid gland failed to reveal any cause. Then the patient was referred to us for definitive management. Taking the history into consideration, a probable diagnosis of Juvenile Recurrent Parotitis due to sialectasis was considered. CT Sialography revealed dilatation of the main duct and the ductules with collection of the dye at the termination of the terminal ductules, in the left parotid gland. Superficial Parotidectomy was considered as the most appropriate management and the patient's parents were counselled for the same. The patient was investigated thoroughly and was posted for Superficial Parotidectomy. The surgery was uneventful and there was no evidence of any postoperative complications. But two days after the surgery, the patient complained to recurrence of swelling and pus discharge from the duct. The patient was started on intravenous antibiotics and the wound was reexplored. During re-exploration, collection of pus was seen in the operated area and palpation of the duct revealed a large sialolith. The pus was drained out and the stone was removed. The patient made a remarkable recovery after this and there has been no evidence of recurrence so far (Figures 1-5). 


\section{Otolaryngology Open Access Journal}
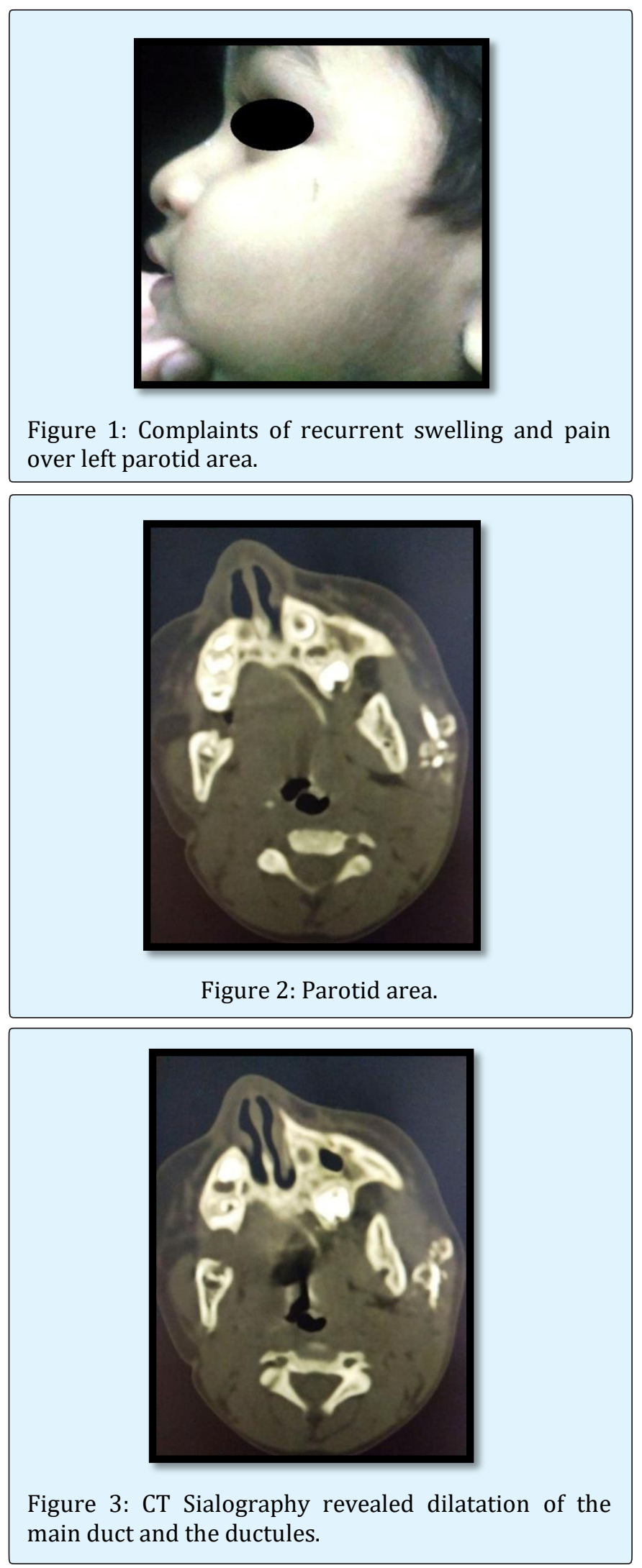
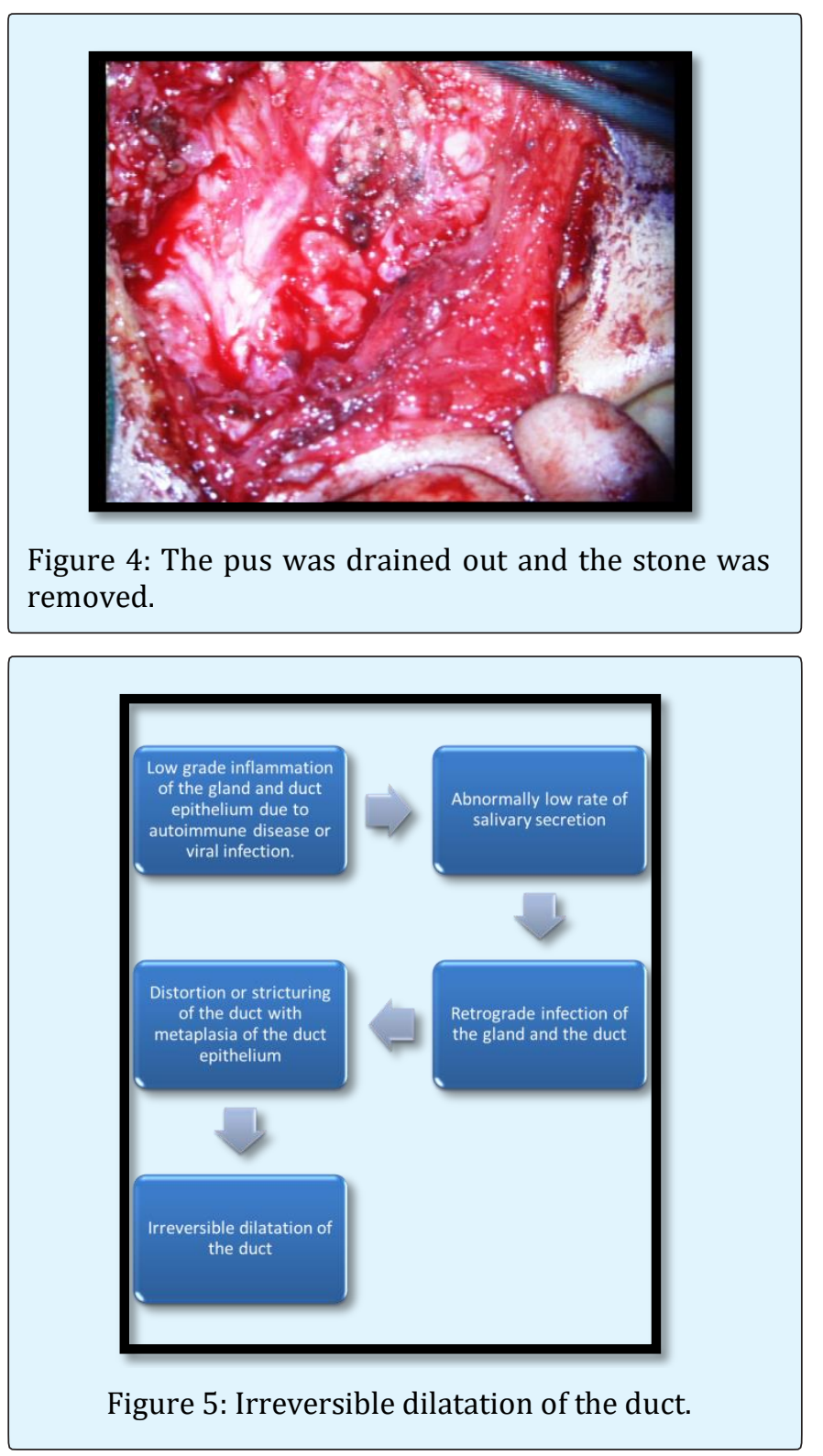

\section{Discussion}

Despite several studies been performed on Juvenile Recurrent Parotitis, the cause still remains unknown. Maynard [1] in 1965 postulated that infections of the oral cavity act the chief predisposing factor and suggested a sequence of events whose end result was recurrent parotid infection. Patey \& Thakray [2] studied the histopathological aspects of the disease and discovered extensive lymphocytic infiltration around the ductules with dilatation of the ducts and formation of cystic cavities. They proposed that lymphocytic infiltrates damage the duct wall reticulum causing extravasation of 


\section{Otolaryngology Open Access Journal}

the secretions into the gland parenchyma and thus, exacerbating the inflammation. This theory was well accepted and was supported by many $[1,3]$. It was further proposed that recurrent infections weaken the connective tissue supporting the intralobular ducts and injection of dye, during sialography, leads to rupture of the ducts and pooling of the dye. This gives the classical appearance of the sialectasis and explains the presence of sialectasis in absence of any demonstrable distal duct obstruction. Hamilton Bailey [4] in 1945, suggested a congenital abnormality of the ductal system as the possible cause and drew a parallel with bronchiectasis [4].

The studies conducted later suggested a multifactorial etiology of this disease. Kono \& Ito [5] suggested that sialectasis was both cause and end result of Juvenile Recurrent Parotitis as the histopathological studies showed areas of true sialectasis and as well as extravasated dye due to damaged ductule walls. Clinical features include swelling over the parotid area and pain during mastication or talking. Males are predominantly affected and most commonly affected age group is 3rd-6th year of life. It is often unilateral but cases of bilateral affliction have been reported. Parotitis is often associated with fever and malaise. There is, typically, absence of pus despite infection. Many predisposing factors have been proposed like immunodeficiency, mumps, allergy, upper respiratory tract infections and autoimmune disorder but none have been conclusively proven.

The gold standard investigation is Sialography. In 1971, Hemenway classified sialectasis into

a) Large duct sialectasis, due to obstruction of the main duct, and

b) Small duct/punctate/terminal sialectasis, in which there are multiple small round opacities at the termination of the smaller ducts [3].

This classification was reviewed and was modified by Gates \& Noyek [6,7] who further classified the sialectasis into

a) pruned tree appearance-in the early stages, the ducts are stretched, tapered, and decreased in number;

b) punctate sialectasis- the peripheral ducts demonstrate punctate dilatation $<1 \mathrm{~mm}$ diameter, and the intraglandularductal system is stretched and tapered;

c) globular sialectasis-the ducts are between $1 \mathrm{~mm}$ and $2 \mathrm{~mm}$ in size and they may be partially non-visualised or irregular, giving rise to a 'mulberry pattern' fruit laden tree appearance;

d) cavitatory sialectasis-coalescence of cystic lesions produces a cavitatory appearance; and e) destructive sialectasis-there is a bizarre pattern of pooling, possibly with stones in the gland. A recent study by Hohmann, et al. [8] has showed that Digital Subtraction Sialographyis superior to conventional for the recognition of inflammatory changes and chronic sialolithiasis.

Recent authors have revealed that Ultrasonography reveals hypoechoic areas which correspond to the punctate sialectasis on Sialography. Since this investigation is less time consuming, easily available and does not require instillation of dye, it is now considered to be the primary screening investigation. On the downside, low sensitivity and operator errors, make it a less favorable choice. Murrat, et al. [9], in 1996, proposed a protocol for investigating a case of intermittent pain and/or swelling of the salivary glands, and they recommend an initial ultrasound. If ultrasound reveals calculi or duct dilatation, then proceed to sialography. In case duct dilatation having been already revealed by ultrasonography, then sialography is required to rule out duct stenosis or obstruction. If the ultrasonography reveals no abnormality, sialography should be performed only if symptoms recur. Biopsy is recommended only in rarest of rare cases and histology usually reveals dilated interlobular ducts with lymphocyte infiltration in the surrounding tissues with the tendency of the lymphocytes to form lymphoid follicles. The duct epithelium shows hyperplasia and metaplasia, with a pseudostratifiedcylindric pattern being common.

Due to unclear etiological factors, an universally accepted treatment for this condition is not yet developed. Treatment of acute episodes usually involves antibiotics, preferably co-amoxyclav, analgesics, antipyretics and anti-inflammatory drugs. Most of the patients usually respond to this treatment and recover. Prevention of recurrence far more challenging and yet important management as it prevents destruction of the gland parenchyma and loss of architecture of the duct walls. Sialogogic agents and massages can be used to increase the secretions and prevent pooling. Prophylactic antibiotics can be used to delay the acute episodes [10].

Treatment of repeated attacks, with proven changes on Sialography, is far more difficult. Over the years many methods have been tried with varying results. Radiotherapy was one of the first methods to be tried for this condition but was soon discontinued as virtually no evidence could be gathered that could reveal that ben efits outweigh the adverse effects. Diamant \& Enfors [11] recommended duct ligation as a simple and effective 


\section{Otolaryngology Open Access Journal}

treatment but this success rate of this method was variable and inconsistent. In 1995, Daud et al. [12] put forth a paper suggesting Tympanic Neurectomy as an effective surgery for parotid sialectasis with success rate of over $70 \%$. This treatment includes sectioning of the secretomotor fibres to the parotid gland that reduce the secreations. Failure of this procedure can be due to incomplete nerve sectioning, causing an early failure or nerve regeneration which causes late failure. Parotidectomy remains the gold standard treatment for such condition but injury to facial nerve remains an insurmountable obstruction, especially in cases of recurrent parotitis resulting in extensive fibrosis of the parenchyma.

\section{Conclusion}

Coexistence of Sialolith and Recurrent Juvenile Parotitsis unheard of. In literature it has been included as a part of the Destructive Sialectasis and has not been reported in studies. As an afterthought, it sounds logical that formation of sialolith should be expected as a consequence of recurrent parotitis due to dystrophic calcification and also recurrent parotitis can be seen in Sialolithisis. Yet reporting of such co-existence has not been a feature of most of the studies. Through this article, we would like to draw attention to such coexistence and encourage further studies in this area.

\section{References}

1. Maynard JD (1965) Recurrent parotid enlargement. Br J Surg 52(10): 784-789.

2. Patey DH, Thakray AC (1955) Chronic 'sialectatic' parotitis in the light of pathological studies on parotidectomy material. Br J Surg 43: 43-50.

3. Hemenway HG (1971) Chronic punctate parotitis. Laryngoscope 81: 485-509.
4. Bailey H (1945) Congenital parotid sialectasis. Journal of the International College of Surgeons 8: 109-114.

5. Kono A, Ito E (1979) A study on the pathogenesis of recurrent parotitis in childhood. Ann Otol Rhinol Laryngol 88(63): 1-20.

6. Gates GA (1977) Sialography and scanning of the salivary glands. Otolaryngol Clin North Am 10(2): 379-390.

7. Noyek A, Zizmor J, Musumeci R, Sanders DE, Renouf JH (1977) The radiologic diagnosis of malignant tumours of the salivary glands. J Otolaryngol 6(5): 381-385.

8. Hohmann D, Landwehr P (1991) Clinical value of sialography in digital and conventional imaging technique. HNO 39(1): 13-17.

9. Murrat ME, Buckenham TM, Joseph AE (1996) The role of ultrasound in screening patients referred for sialography: a possible protocol. Clin Otolaryngol Allied Sci 21(1): 21-23.

10. Ericson S, Zetterlund B, Ohman J (1991) Recurrent parotitis and sialectasis in childhood. Clinical, radiologic, immunologic, bacteriologic, and histologic study. Ann Otol Rhinol Laryngol 100: 527-535.

11. Diamant H, Enfors B (1965) The treatment of chronic recurrent parotitis. Laryngoscope 75: 2644.

12. Daud AS, Pahor AL (1995) Tympanic neurectomy in the management of parotid sialectasis. J Laryngol Otol 109(12): 1155-1158. 